\title{
THE TROUBLE WITH RESONANCE ENERGIES: A HÜCKEL THEORY TOPIC
}

\author{
Richard Francis Langler \\ Department of Chemistry, Mount Allison University, Sackville, New Brunswick E4L 1G8 Canada
}

Recebido em 4/5/04; aceito em 1/2/05; publicado na web em 30/6/05

\begin{abstract}
Resonance energies are shown to be quasithermodynamic in character. Hence, they are generally unsuitable as bases for anticipating kinetic stabilities. Examples are provided, leading to the conclusion that those who intend the word 'aromatic' to mean chemically unreactive, need to carry out full Hückel calculations in order to rank hydrocarbons using the frontier orbital energies.
\end{abstract}

Keywords: aromaticity; kinetic stability; resonance energies.

\section{INTRODUCTION}

Benzene has long been recognized as an especially stable molecule which serves as the best example of an aromatic $\pi$ system. Indeed, in 1866, Erlenmeyer proposed that the word aromatic be applied to molecules which are related to benzene in a chemical sense i.e. low reactivity (high stability) and a preference for substitution reactions rather than addition reactions ${ }^{1}$.

Because the word stable has two rather different meanings, a good deal of ambiguity may attend its use in chemical discussions. Stable, in the thermodynamic sense, relates a given structure to the energy gain/loss when isolated atoms are joined to produce the structure in question. Stable, in the kinetic sense, characterizes a structure in terms of its tendency to react with other molecules. A compound is particularly unstable, in the kinetic sense, when it will react with itself, at or below ambient temperature e.g. cyclobutadiene (see Scheme 1).

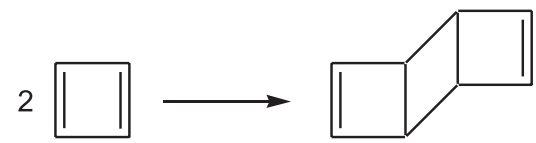

Scheme 1. Facile dimerization of cyclobutadiene

Haddon and Fukunaga ${ }^{2}$ have shown a direct connection between kinetic and thermodynamic stabilities for the annulenes e.g. cyclobutadiene and benzene. Hence, for the annulenes, enhanced thermodynamic stability implies enhanced kinetic stability.

A variety of resonance energies have been defined and proposed, at the Hückel level, to organize and facilitate the classification of hydrocarbons as aromatic, nonaromatic or antiaromatic. Further discussion will use Dewar resonance energies ${ }^{3,4}$ to represent such energies. At the Hückel level, Dewar resonance energies are obtained by calculating total $\pi$ electronic stabilization $\left(\mathrm{E}_{\pi}\right)$ for the cyclic molecule of interest. Thereafter, the $\mathrm{E}_{\pi}$ for the corresponding unbranched, isoconjugate hydrocarbon is obtained. The difference between these $\mathrm{E}_{\pi}$ values is the Dewar resonance energy. As an example, the Dewar resonance energy (DRE) for benzene is calculated in Figure 1.

When a Dewar resonance energy is positive, the compound is aromatic, when a Dewar resonance energy is zero the compound is

*e-mail: rlangler@mta.ca nonaromatic and when a Dewar resonance energy is negative the compound is antiaromatic.

Since Dewar resonance energies use $\mathrm{E}_{\pi}$ values which account for the stabilization of all $\pi$ electrons, they represent Hückel level numbers which are thermodynamic in character. However, Hückel descriptions are severly limited e.g. they do not account for steric crowding or ring strain. Hence, Hückel resonance energies are quasithermodynamic.

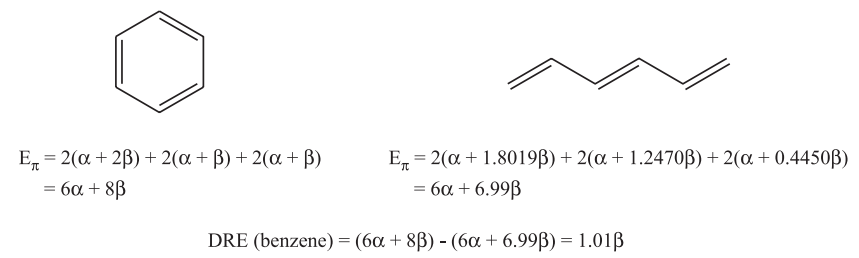

Figure 1. Calculation of the Dewar resonance energy for benzene

In contrast, kinetic stability primarily depends upon the relative energies of the frontier molecular orbitals i.e. the HOMO energy correlates with nucleophilic/basic behaviour and the LUMO energy correlates with electrophilic behaviour ${ }^{5}$. The following section examines structural connections between conflicting quasithermodynamic/kinetic stabilities for hydrocarbons.

Note that the relative order of Hückel eigenvalues, for a particular molecule, may not be reliable and cannot be used to predict electronic spectra.

\section{RESULTS AND DISCUSSION}

The best known Hückel descriptions for a series of hydrocarbons are those of the annulenes. Because each $\mathrm{C}_{2 \mathrm{n}}$ annulene has a plane of symmetry bisecting it into noninteracting segments, each even annulene has a set of antisymmetric Hückel orbitals which have the energies (eigenvalues) associated with the unbranched $\mathrm{C}_{\mathrm{n}-1}$ acyclic polyene. Using planar cyclooctatetraene as an example, one can see that it must have eigenvalues associated with the allyl group (see Figure 2).

Because each even annulene has a principal symmetry axis of order $\geq 3$, the eigenvalues deduced from a symmetry argument (illustrated above) occur in degenerate pairs. This, of course, is exactly what is anticipated from the Frost-Musulin circle mnemonic ${ }^{6}$ (see Figure 3).

There is a class of less familiar hydrocarbons for which a simi- 


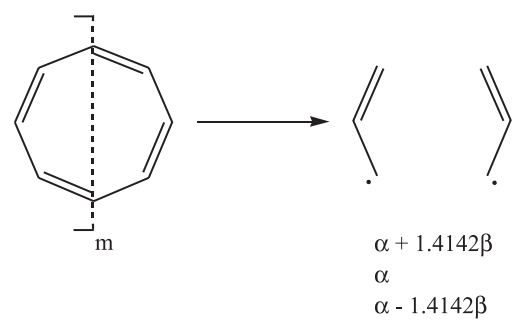

Figure 2. Symmetry-based deduction that cyclooctatetraene has Hückel molecular orbitals with the allyl eigenvalues

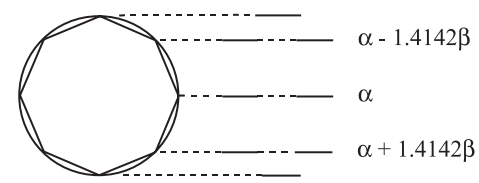

Figure 3. The Frost-Musulin circle mnemonic applied to cyclooctatetraene

lar analysis leads to related descriptions. A general form for these compounds is shown as $\mathbf{1}$.<smiles>CCC(CC)CC</smiles>

1

Since Hückel descriptions are insensitive to distortions of the $\sigma$ framework, compounds $\mathbf{1}$ have Hückel descriptions that are unaffected by imposing a $\mathrm{Y}$ shape on those structures. Hence, the Hückel descriptions impose a virtual symmetry plane which bisects the $\mathrm{Y}$ shape. Thus, these branched, acyclic $\mathrm{C}_{4+3 \mathrm{x}}$ polyenes have a set of antisymmetric $\pi$-orbitals which have the eigenvalues associated with unbranched, acyclic $x+1$ polyenes. Using the $C_{10}$ case $(1, x=2)$ as an example, one can see that it must have the eigenvalues associated with the allyl group (see Figure 4).

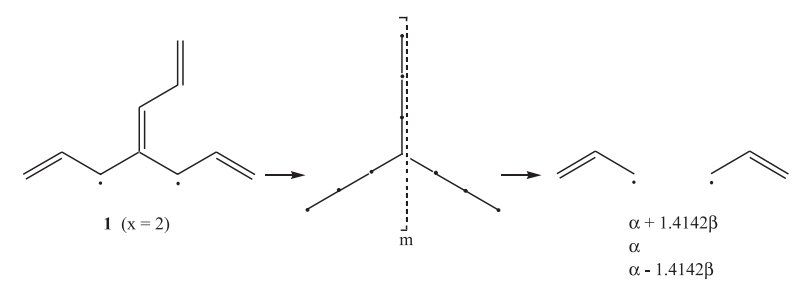

Figure 4. Virtual symmetry-based deduction that the branched polyene $\mathbf{1}$ $(x=2)$ has Hückel $\pi$-orbitals with allyl eigenvalues

Because each Y shaped structure $\mathbf{1}$ has a principal symmetry axis of order 3 , the eigenvalues deduced from a symmetry argument (illustrated above) occur in degenerate pairs. The annulenes and the acyclic, branched polyenes, $\mathbf{1}$, can be grouped into pairs, by carbon content, where the annulene has $4+2 \mathrm{~N}$ carbon atoms and the acyclic polyene has $4+3 \mathrm{~N}$ carbon atoms. Each pair of structures will share common degenerate pairs of eigenvalues e.g. the $\mathrm{C}_{8}$ annulene $\left(\mathrm{C}_{4+2(2)}\right)$ and the $\mathrm{C}_{10}$ branched polyene $\left(\mathrm{C}_{4+3(2)}\right)$ each have a pair of eigenvalues at $\alpha+1.4142 \beta$, another pair at $\alpha$ and another pair at $\alpha-1.4142 \beta$.

From the foregoing arguments, it follows that benzene and $\mathbf{1}(x=1)$ each have a degenerate pair of bonding orbitals at $E=\alpha+\beta$.
Fortuitously, they each have a unique orbital at $E=\alpha+2 \beta$. Hence, the secular equation for $\mathbf{1}(\mathrm{x}=1)$ is the secular equation for benzene multiplied by $\mathrm{x}$. At the Hückel level, the cation derived from $\mathbf{1}(\mathrm{x}=1)$ and benzene have identical $\pi$ electron stabilizations $(E \pi=6 \alpha+8 \beta)$. If one chose to describe these isoelectronic structures as aromatic in a quasi-thermodynamic sense, would one expect $\mathbf{1}(x=1)^{+}$to be aromatic in a kinetic sense?

$\mathbf{1}(\mathrm{x}=1)^{+}$, in sharp contrast to benzene, is expected to be a very reactive electrophile $\left(\mathrm{E}_{\mathrm{LUMO}}=\alpha\right)$ and therefore, not kinetically stable. Application of the word aromatic to $1(x=1)^{+}$would be entirely inappropriate for anyone who, like Erlemneyer, would like to associate kinetic stability with it.

There is nothing special, at the Hückel level, about carbocations that have strongly-stabilized $\pi$-electrons but which are kinetically unstable. Any structure for which the HOMO and the LUMO are unsymmetrically arranged about the nonbonding level, will have enhanced electrophilicity if the LUMO is closer to the nonbonding level $(\alpha)$ than the HOMO is. This is a commonplace amongst nonalternant hydrocarbons. As an example, although pentalene 2 has a positive Dewar resonance energy $(\mathrm{DRE}=0.94 \beta)$ and may be considered aromatic on a quasithermodynamic basis, it is a very reactive electrophile. This is correctly anticipated by the Hückel description which provides a nonbonding LUMO $\left(\mathrm{E}_{\mathrm{LUMO}}=\alpha\right)$ for 2 .

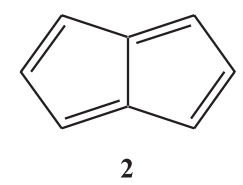

For nonalternant hydrocarbons, $\alpha$ is not a limit for LUMO stabilization. In many structures, the LUMO can be significantly more strongly stabilized ${ }^{7}$. As an example, the Hückel LUMO for $\mathbf{3}$ is bonding $\left(\mathrm{E}_{\mathrm{LUMO}}=\alpha+0.2451 \beta\right)$.

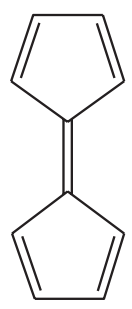

3

Neither does $\alpha$ serve as a limiting energy for HOMO destabilization $^{7} .4$ provides an example for which the HOMO is antibonding $\left(\mathrm{E}_{\text {номо }}=\alpha-0.4142 \beta\right)$.

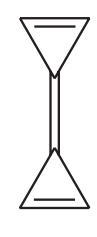

4

From the kinetic stability standpoint, both $\mathbf{3}$ and $\mathbf{4}$ would be very reactive and so best viewed as antiaromatic. From the quasithermodynamic standpoint, $\mathbf{3}$, which has strongly stabilized $\pi$ electrons, should be aromatic and is so characterized by its Dewar resonance energy $(3, \mathrm{DRE}=0.75 \beta)$. Given the Hückel level prediction that $\mathbf{4}$ should have antibonding electrons, it may be 
surprising that it is classified as aromatic by its Dewar resonance energy $(4, \mathrm{DRE}=0.48 \beta)$. In contrast, the topological resonance energy (per electron) $)^{8}$ for 4 is $-0.076 \beta$ which puts it in the antiaromatic category $(\mathrm{TRE}(\mathrm{PE})<-0.01 \beta)$ on that basis. Note that topological resonance energies have major problems handling Lewis structures which must show nonbonding electrons ${ }^{8}$.

Resonance energies lead to inappropriate kinetic stability conclusions for many pairs of alternant hydrocarbons as well. Consider the $\mathrm{C}_{14}$ alternant hydrocarbons 5 and $\mathbf{6}$ (see Figure 5).

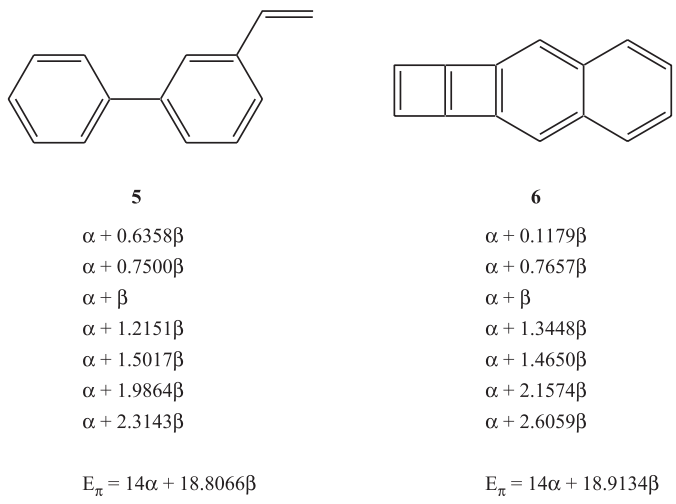

Figure 5. Eigenvalues and $E_{\pi}$ values for the isoconjugate hydrocarbons 5 and 6

From the Hückel results given in Figure 5, 6 would be the more aromatic compound (based on $\mathrm{E}_{\pi}$ values) in a quasithermodynamic sense. In sharp contrast, the frontier electron stabilization for $\mathbf{5}$ is more than five times better than that for $\mathbf{6}$, leading to the conclusion that $\mathbf{5}$ would be a much less reactive nucleophile than $\mathbf{6}$ and hence a great deal more aromatic in a kinetic sense. Note that both $\mathbf{5}$ and $\mathbf{6}$ are alternant hydrocarbons, hence $\mathbf{5}$ is a much less reactive electrophile $\left(\mathrm{E}_{\mathrm{LuMO}}=\alpha-0.6358 \beta\right)$ than $6\left(\mathrm{E}_{\text {LuмO }}=\alpha-0.1179 \beta\right)$ as well. The origin of the kinetic/quasithermodynamic stability reversal is that $\mathbf{5}$, which has the more strongly stabilized frontier electrons, has generally more weakly stabilized inner electrons, particularly those in the lowest lying orbitals, $\Psi_{1}$ and $\Psi_{2}$.

\section{CONCLUSIONS}

Although Hückel theory can be helpful for a range of structural and behavioural problems associated with organic molecules ${ }^{9-15}$, the notion of resonance energies has been troublesome.

At the Hückel level of theory, resonance energies are generally quasithermodynamic in character. Hence, the word aromatic, when applied on the basis of calculated resonance energies need not, and often will not, correlate with expected kinetic stability for a given structure. The fact that quasithermodynamic and kinetic stabilities, obtained from the application of Hückel theory to hydrocarbon $\pi$ systems, often conflict does not reveal a flaw in Hückel theory. For those who would assess hydrocarbon aromaticity on the basis of anticipated kinetic stability, full Hückel calculations need be done to establish the eigenvalues for the frontier orbitals. It would be helpful indeed, if a straightforward "back of the envelope" method were available to rank hydrocarbons on the basis of their frontier orbital eigenvalues. Then, Erlenmeyer's proposal of 1866 could finally be placed on a utilitarian theoretical basis.

\section{REFERENCES}

1. Snyder, J. P. In Nonbenzenoid Aromatics; Snyder, J. P., ed.; Academic Press: New York, 1969, p. 14

2. Haddon, R. C.; Fukunaga, T.; Tetrahedron Lett. 1980, 21, 1191.

3. Dewar, M. J. S.; Dougherty, R. C.; The PMO Theory of Organic Chemistry, Plenum Press: New York, 1975.

4. Durkin, K. A.; Langler, R. F.; J. Phys. Chem. 1987, 91, 2422.

5. Fleming, I.; Frontier Orbitals and Organic Chemical Reactions, Wiley: Chichester, 1976, p. 23-32.

6. Frost, A. A.; Musulin, B. J.; J. Phys. Chem. 1953, $21,572$.

7. Langler, R. F.; Aust. J. Chem. 2002, 55, 715.

8. Langler, R. F.; Aust. J. Chem. 1991, 44, 297 and references therein.

9. Langler, R. F.; J. Chem. Educ. 1996, 73, 899

10. Langler, R. F.; Chem. Educator 1998, 3, S1430 - 4171 (98) 02 190-6.

11. Langler, R. F.; Salgado, G.; Mendizabal, C.; Quim. Nova 2000, 23, 841.

12. Langler, R. F.; Quim. Nova 2000, 23, 703.

13. Langler, R. F.; Chem. Educator 2000, 5, 171

14. Langler, R. F.; Quim. Nova 2001, 24, 416.

15. Langler, R. F.; Chem. Educator 2002, 7, 1. 